IdeAs

Idées d'Amériques

$17 \mid 2021$

Villes et culture dans les Amériques

\title{
Arte y Cultura en la transformación de barrios populares en América Latina
}

L'art et culture dans la transformation des quartiers populaires d'Amérique latine

The role of Art and Culture in the transformation of poor neighborhoods in Latin America

\section{Luis Campos y Catherine Paquette}

\section{OpenEdition}

\section{Journals}

Edición electrónica

URL: https://journals.openedition.org/ideas/10789

DOI: 10.4000/ideas.10789

ISSN: 1950-5701

Editor

Institut des Amériques

Referencia electrónica

Luis Campos y Catherine Paquette, «Arte y Cultura en la transformación de barrios populares en

América Latina», IdeAs [En línea], 17 | 2021, Publicado el 01 marzo 2021, consultado el 03 junio 2021 URL: http://journals.openedition.org/ideas/10789 ; DOI: https://doi.org/10.4000/ideas.10789

Este documento fue generado automáticamente el 3 junio 2021.

\section{$(\mathbb{A} \otimes \Theta$}

IdeAs - Idées d'Amériques est mis à disposition selon les termes de la licence Creative Commons Attribution - Pas d'Utilisation Commerciale - Pas de Modification 4.0 International. 


\section{Arte y Cultura en la transformación de barrios populares en América Latina}

L'art et culture dans la transformation des quartiers populaires d'Amérique

latine

The role of Art and Culture in the transformation of poor neighborhoods in Latin America

Luis Campos y Catherine Paquette

\section{Introducción}

1 América Latina posee una tradición y un saber-hacer importantes en materia de programas públicos de mejoramiento urbano de barrios marginados (Rojas E., 2009; Orozco Martínez Y., 2015). Desde hace por lo menos tres décadas, en esta región muy urbanizada que se caracteriza por haber integrado plenamente la informalidad del desarrollo humano (a diferencia de otras regiones del Sur Global) se han llevado a cabo esfuerzos sostenidos para mejorar un sin número de asentamientos humanos, productos del proceso de urbanización informal explosivo que se dio durante la segunda mitad del siglo XX y en especial hasta los años 80 .

2 Estos programas han conocido una evolución notable a lo largo de tiempo, pasando de ser iniciativas orientadas en una primera instancia ante todo hacia la provisión de infraestructuras vinculadas a la dotación en servicios urbanos básicos (agua, energía, saneamiento) y al mejoramiento habitacional, a enfoques cada vez más multisectoriales, que ponen mucho énfasis en la participación ciudadana y la coproducción de los proyectos con la población residente, a modo de impulsar la integración urbana, social y simbólica de las comunidades y los individuos. De hecho, estos programas, cuya gran diversidad es de destacar, constituyen en la actualidad una herramienta predilecta para reducir las desigualdades socio-espaciales en las ciudades 
de la región, a tal punto que hoy en día se está apostando a aquellos como una estrategia de recuperación post-pandemia de largo plazo, basada en el bienestar de la población, la concreción de sus derechos y el respeto del entorno ${ }^{1}$.

El uso del arte y de la cultura en general ha cobrado una importancia creciente en numerosos programas de mejoramiento de barrios impulsados desde los gobiernos nacionales o locales. Se llegó inclusive a considerar estas dimensiones como un pilar central en las estrategias de transformación urbana de asentamientos marginados, como en el caso emblemático del urbanismo social de Medellín. La organización de talleres artísticos, la realización de murales por parte de los habitantes de los barrios (en particular los jóvenes), así como la instalación de casas de la cultura, bibliotecas y otros faros del saber forman parte del conjunto de acciones que están siendo implementadas con éxito en numerosos barrios de origen informal, así como ahora en conjuntos de vivienda social deteriorados en proceso de regeneración (problemática urbana emergente en varios países de la región). No obstante, no existe hasta la fecha un proceso de estudio y evaluación sistemáticos de estos impactos y tampoco una conceptualización detallada de este tipo de intervención basada en el uso del arte y de la cultura para contribuir al mejoramiento barrial.

4 Si bien es cierto que el hecho de recurrir al arte y a la cultura desde el Urbanismo, para generar una transformación urbana, se ha vuelto muy común desde hace ya varias décadas, tanto en Europa y América del Norte como en América Latina, nos encontramos aquí ante algo distinto. En efecto, no se trata de impulsar grandes proyectos de regeneración en áreas urbanas valorizadas (centros históricos, zonas centrales codiciadas) o en proceso de valorización (áreas de baldíos en proceso de recuperación por parte de las autoridades o del sector inmobiliario), aprovechando la construcción de grandes equipamientos culturales, como son los "museos monumentales" (Lusso B., 2009) o la organización de grandes eventos ligados a la cultura (Masboungi A., 2011). Tampoco nos estamos refiriendo a iniciativas más puntuales y acotadas de urbanismo transitorio y temporario, ahora muy en boga, que recurren a colectivos de artistas para acompañar la transición urbanística en barrios en proceso de regeneración (Desgouttes, 2019). Todas estas intervenciones urbanísticas a escalas diversas, por lo general muy vinculadas a procesos de gentrificación subyacentes, buscan desarrollar atractivos territoriales para captar nuevos residentes, usuarios o inversores a través de una transformación de la imagen urbana.

5 En cambio, los programas de mejoramiento de barrios marginados se caracterizan por estar orientados a producir un desarrollo endógeno de los barrios, que permita mejorar las condiciones de vida y la integración urbana y social ante todo de las poblaciones residentes, las que están estrechamente involucradas en el proceso. Las experiencias de mejoramiento de barrios llevadas a cabo en América Latina constituirían en este sentido una forma de regeneración urbana diferente y alternativa, en la cual la región tendría una experiencia avanzada respecto a otras partes del mundo (Paquette C., 2020). En aquellas, debido a los objetivos perseguidos, el uso del Arte y la Cultura distaría mucho de lo que suele existir en el contexto de las estrategias urbanas de valorización, orientadas hacia la competitividad urbana y la creación de "marca ciudad" (Zamorano M., 2020). Se estaría buscando más bien generar dinámicas comunitarias que permitan fomentar la inclusión de los barrios y sus habitantes a la ciudad, detonador de un proceso de mejoramiento urbano y social. 
6 Aunque resulta importante reconocer que también existen experiencias de la misma índole en economías desarrolladas, en las que se recurre al arte y la cultura para contribuir a la transformación de barrios marginados ${ }^{2}$, es preciso subrayar que estos proyectos no llegan a formar parte de los elementos centrales de las estrategias urbanas implementadas, a diferencia de lo que sucede en América Latina. A modo de ejemplo, en Francia, uno de los países que se encuentra llevando a cabo desde hace ya varias décadas los programas de regeneración de barrios de vivienda social más ambiciosos a nivel internacional, existen por cierto experiencias en la materia y las autoridades inclusive recomiendan desde hace tiempo vincular el arte y la cultura al rescate de los espacios públicos (Auclair, E., 2006). Sin embargo, no se trata de una tendencia generalizada o en proceso de generalización. Al contrario, desde hace dos décadas, los programas públicos para regenerar los grandes conjuntos habitacionales en crisis se han volcado claramente hacia acciones de restructuración físico-espacial mayores, adoptando un enfoque definitivamente urbanístico (rehabilitaciones físicas, demoliciones, construcción nueva, re-estructuración profunda del tejido urbano) y no social, apostando a este tipo de transformación estructural para mejorar los barrios que se encuentran en situación crítica desde un punto de vista físico, social y económico (Bellanger E. et al., 2018).

7 Atendiendo a estos elementos principales, el objetivo de este artículo es reflexionar acerca de los rasgos característicos del uso del arte y la cultura en los programas de mejoramiento de barrios marginados de América Latina, para así entender la especificad del enfoque que materializan hacia la problemática que buscan atender. Argumentamos que el elemento distintivo de estos proyectos radica en el uso del arte y la cultura como agente detonador de la integración social de los habitantes de los barrios, a través de iniciativas que buscan activar y mantener su participación permanente y así, indirectamente, provocar transformaciones en el tejido urbano.

8 Sin embargo, podemos considerar que aún no se ha avanzado en desarrollar intervenciones en la materia que sean articuladas territorialmente, ni en establecer procedimientos de estimación de la incidencia de los proyectos implementados en materia de arte y cultura en los barrios. Por lo cual otro propósito del artículo es abrir la reflexión al respecto y sentar las bases para una agenda de investigación acerca de esta temática, hasta el momento poco explorada, a pesar de la relevancia que posee en las estrategias de mejoramiento de barrios marginados de América Latina.

\section{El arte y la cultura en las iniciativas de transformación urbana}

9 Desde hace ya varias décadas, el arte y la cultura se han convertido en herramientas fundamentales para programas, proyectos y políticas de intervención urbana en el mundo (Duque I., 2015; UN-Habitat, 2004; UNESCO, 2001). A pesar de que los campos de actuación han sido variados y han incluido desde la intervención sobre centros históricos deteriorados, hasta la articulación de clusters urbanos de industrias culturales, pasando por el rediseño de espacios públicos y la adaptación a los requerimientos del turismo, ha predominado un tipo de acción que busca producir una nueva imagen de ciudad, destacando su singularidad y sus ventajas competitivas como estrategia de diferenciación respecto de sus rivales globales (Duque I. 2015; RiusUlldemolins J. y L. Posso Jiménez, 2016). Es lo que podemos llamar la cultura como 
estrategia de competitividad urbana y de creación de "marca ciudad" (Zamorano M., 2020).

Sin embargo, esta descripción general esconde importantes matices a la hora de analizar las formas en que se ha concebido y conceptualizado la cultura en las intervenciones urbanas, así como el tipo de actuaciones específicas que se ha desplegado en su nombre, en distintas zonas del mundo. Dicho de otra forma, tal descripción no permite entender las diversas racionalidades detrás del uso de la cultura en las intervenciones urbanas (Zamorano M., 2020: 92), como tampoco permite apreciar la variedad de repertorios de intervención que se ha utilizado en cada región.

11 Siguiendo la distinción analítica planteada por Duque (2015), resulta plausible proponer que, además de la cultura como estrategia de competitividad urbana, en las intervenciones urbanísticas también aparece "la cultura asociada al comportamiento, es decir, la cultura ciudadana; la cultura como una dimensión de la ciudad a escala humana que se traduce en equipamientos y espacio público; la cultura desde la perspectiva de los derechos civiles, económicos, sociales y culturales" (Duque I., 2015: 28-9).

12 Esa variedad de enfoques redunda en que, aparte de los proyectos de city branding (Zamorano M., 2020), exista una diversidad de iniciativas que utilizan la cultura como estrategia de transformación urbana y a una diversidad de escalas. Así, es posible identificar intervenciones al estilo del "Modelo Barcelona" que, como indican Rius y Sánchez-Belando (2015), ha implicado una estrecha relación entre el modelo de desarrollo local y el de política cultural y que, en los últimos años, ha experimentado una creciente instrumentalización en favor del desarrollo económico y la proyección internacional. Pero también es posible observar intervenciones del llamado "urbanismo táctico" a través de las cuales se busca la recuperación de espacios públicos y que son entendidas como "acciones de corto plazo que buscan generar un cambio de largo plazo en la forma de habitar la ciudad"3, en la medida en que debido a su rápida ejecución y bajo costo pueden ser realizadas con mucha rapidez y se puede poner a prueba su impacto para modificarlas a la luz de los resultados obtenidos (Douay N. y M. Prévot, 2016).

13 Tras el empeño por efectuar un balance para el caso europeo, García (2008) ha planteado que el tratamiento dado a la cultura en las iniciativas de regeneración desplegadas en Europa occidental se caracteriza por una tendencia a considerar a la cultura " "solamente en términos funcionales que dan prioridad a la pregunta: "qué puede aportar la cultura a la economía” en lugar de permitir el lanzamiento de los avances sociales y culturales y reconocer su valor intrínseco para la regeneración urbana" (p.113). Esta convergencia entre cultura y economía ha derivado en la acentuación de "las técnicas de marketing urbano y su progresiva transformación en estrategias de creación de marcas urbanas" (p.114), pero también en que "la mayoría de estas infraestructuras han sido esencialmente diseñadas como emblemas de prestigio, lo que ha conseguido, en términos generales, promocionar la imagen de la ciudad y atraer el turismo, pero ignorando las necesidades sociales y culturales de la comunidad local y teniendo un impacto limitado sobre las tasas de empleo y la recuperación económica de la zona a largo plazo" (p.119).

14 Es en esta diversidad de posibilidades donde conviene situar a los Programas de Mejoramiento de Barrios (PMB), desplegados en América Latina desde hace más de tres décadas, muchos de ellos bajo el auspicio financiero del Banco Interamericano de 
Desarrollo. En efecto, como señalan Marengo y Elorza, en "el plano sectorial de la política social, especialmente el de hábitat, [se ha promovido] programas tendientes a mitigar el crecimiento de la pobreza, la inseguridad y la violencia urbana. A partir de los años noventa, el hábitat informal urbano se convirtió en una línea prioritaria de intervención y financiamiento (2010: 80). Las mismas autoras hablan de una nueva "generación de políticas" habitacionales que "se traduce en distintos programas en la región: Favela Bairro en Brasil, Desmarginalizacion de la Alcaldía de Bogotá, Programa de Mejoramiento Barrial en Argentina, entre otros, que buscan promover una mejor calidad de vida de los pobladores de asentamientos informales a través de la ejecución de obras públicas, mejoramiento de vivienda y regularización dominial" (Marengo C. y A. Elorza, 2010: 81).

15 Los PMB han buscado abordar los problemas de deterioro y desintegración, en los planos físico, económico y sociocultural, teniendo la doble finalidad de intervenir sobre el tejido social y recuperar espacios deteriorados, haciendo resonar los planteamientos de Gino Germani (1967, citado en Ziccardi A., 2012), quien señalaba que la ciudad constituía un mecanismo de integración social en dos sentidos: a) la integración geográfica a la sociedad, y b) la integración social vinculada a la participación, la movilización y la marginalidad, entendida esta última desde una visión ecológica y psicosocial (Giglia A., 2016). En ese sentido, los PMB operan con una concepción de cultura más cercana al comportamiento y a la vida ciudadana -de acuerdo a los planteamientos de Duque (2015) antes indicados- o a lo que la UNESCO (1982) ha considerado la cultura como la forma organizada de la vida social, inspirada en una visión de mundo que se expresa en un conjunto de conductas y pensamientos aceptados por el colectivo.

Los PMB conciben los espacios públicos como el elemento articulador de la vida urbana y un agente fundamental en la promoción del acceso, participación e inclusión de la población más vulnerable. Así, haciendo eco de planteamientos como el de la Nueva Agenda Urbana adoptada en 2016 a raíz de la conferencia mundial Hábitat III, presumen que dichos espacios constituyen un bien común y tienen la capacidad de generar oportunidades para grupos postergados como mujeres, jóvenes y adultos mayores, y se erigen como una poderosa herramienta en la lucha contra la discriminación (ONU, 2017). Y aunque las intervenciones parecen asumir que el espacio público en las ciudades latinoamericanas generalmente no posee esos atributos -en la medida que es el resultado de ciudades que se han construido con déficits de planificación, con mucha fragmentariedad y con predominio de la autoconstrucción-, se apoyan en la convicción de que la población que habita en estos barrios tiene capacidad de resistencia a la adversidad y ha desarrollado un repertorio cultural con fuerte identidad y capacidad transformadora.

17 Para alcanzar sus objetivos, los PMB han convertido a las acciones culturales y artísticas en importantes herramientas de intervención, verdaderos productores de integración social y de resignificación espacial de lugares situados al margen, sin las condiciones necesarias para una vida adecuada. De hecho, tales programas despliegan intervenciones culturales como acciones destinadas a facilitar la apropiación colectiva del entorno próximo, pero también para intervenir en lugares peligrosos y degradados, para enfrentar diversas formas de violencia -desde las más visibles, como las ligadas al crimen y el narcotráfico, hasta las más invisibles y estructurales, como las violencias 
cotidianas que sufren las mujeres-, así como para cohesionar comunidades desarticuladas.

Entre las intervenciones artísticas que despliegan los PMB tiene un rol fundamental la elaboración colectiva y participativa de murales, mosaicos y otras formas de arte visual, pero también es posible encontrar otro tipo de acciones, ligadas a la música y la danza, tales como la organización de bandas musicales, coros y grupos de baile. A pesar de su gran diversidad, estas intervenciones culturales comparten la vocación de reorientar el uso del tiempo libre entre la población más joven, activar la participación de las comunidades locales, aumentar la legitimidad de la acción pública, gatillar procesos de dinamización económica de los barrios, mejorar el estado de conservación de las infraestructuras y equipamientos, y cambiar la imagen pública del lugar.

Así, si se piensa en un ejemplo hipotético de intervención de murales en fachadas de inmuebles, es posible conjeturar que con ella se busca atraer a las y los habitantes jóvenes del barrio, para enrolarlos en un proceso de formación en técnicas y confección de obras que les permita ocupar su tiempo en actividades distintas de las habituales en el barrio; luego, se busca el embellecimiento de fachadas, la mejora de los espacios públicos barriales y el cambio del paisaje local, lo que, a su vez, tiene como propósito atraer visitantes de otras zonas que, con su presencia reiterada, dinamicen la actividad comercial del sector. Todo ello suele realizarse en alianza con otro tipo de intervenciones, como las ligadas al transporte público local, o con actores locales asentados y bien articulados con la comunidad, como son las escuelas, las infraestructuras de salud o, inclusive, las instituciones religiosas e iglesias.

20 A través de este ejemplo hipotético queremos poner en evidencia que la intencionalidad de las intervenciones culturales de los PMB es la de producir una transformación que no sólo involucre el componente físico y material de los barrios, sino que también sus elementos subjetivos, identitarios y de sociabilidad, de manera que se produzca un cambio significativo en la dinámica relacional del barrio.

21 Cabe entonces plantear que la inclusión del componente cultural en las iniciativas desplegadas por los PMB responde a un momento particular de la dinámica urbana y social de las ciudades latinoamericanas que, del lado de la intervención pública, se caracteriza por la emergencia y progresiva institucionalización de los objetivos de integralidad de la intervención y bienestar subjetivo, así como del tránsito de una visión acotada de la necesidad de la provisión de servicios urbanos básicos para el mejoramiento de barrios, hacia un enfoque que abarque otras dimensiones esenciales para la integración plena de los habitantes a la ciudad, como es el acceso a la cultura y, a través de ella, a la ciudadanía. Estos objetivos ampliados implican el diseño, elaboración e implementación de instrumentos de intervención que permitan actuar sobre los elementos físicos y materiales de los barrios, pero también y sobre todo, sobre los aspectos subjetivos de las y los habitantes de los barrios, a los que las iniciativas artísticas resultan afines.

\section{Usos del arte y la cultura en los PMB}

La literatura sobre los programas y proyectos de mejoramiento de barrios en América Latina puede ser considerada como bastante escasa en relación al gran número de acciones de este tipo que han sido y están siendo implementadas en la región. Destaca en particular la relativa ausencia de reflexiones orientadas a teorizar y sistematizar 
este enfoque hacia la regeneración de barrios marginados. Asimismo, los trabajos existentes que buscan proponer un panorama de estas experiencias en la región no suelen abordar el rol del arte y la cultura en dichas políticas, programas y proyectos (Rojas E., 2009; Orozco Martínez Y., 2015). Cabe señalar que las mayores fuentes de información en la materia son finalmente las consultorías, organizaciones de la sociedad civil o iniciativas de investigación-acción, con las que se obtiene información más abundante sobre los usos del arte y la cultura en los PMB 5 . Por ello, para desplegar este análisis se ha debido revisar la literatura referida a estos últimos y, a partir de ella, desentrañar referencias al uso de estos elementos en cada programa. Se presentan a continuación los detalles de una pequeña muestra de programas de mejoramiento de barrios, enfatizando sobre sus componentes en materia de arte y cultura.

Probablemente el ejemplo más conocido a nivel mundial es el proyecto transformador de barrios populares marginados iniciado en Medellín, segunda ciudad de Colombia, a partir de principios de la década del 2000 y ahora conocido como Urbanismo Social. Originalmente se inició en la zona nororiental de la ciudad, y progresivamente se fue extendiendo a otros barrios marginados de la misma, siempre mediante la metodología original de los Proyectos Urbanos Integrales (PUI), instrumentos de planeación e intervención física para los polígonos seleccionados. Dentro de las numerosas acciones que se implementaron destacan, además de las ahora muy conocidas líneas de teleférico urbano (metrocables) para mejorar la movilidad los habitantes y la accesibilidad de las zonas intervenidas, la construcción en los barrios de vastos espacios dedicados a la cultura en un sentido amplio. Es así como fueron desarrollados 9 "parques bibliotecas", con la característica de contar con una arquitectura cuidada y bastante destacable en el paisaje urbano barrial, así como la de estar concebidos para poder albergar una gama muy amplia de actividades culturales, artísticas y también comunitarias y vecinales. El ejemplo del Parque biblioteca San Javier, ubicado en la comuna 13 de Medellín, uno de los territorios más estigmatizados de la ciudad, es uno de los más emblemáticos. 
Figura 1. Interior de la biblioteca San Javier, Comuna 13, Medellín

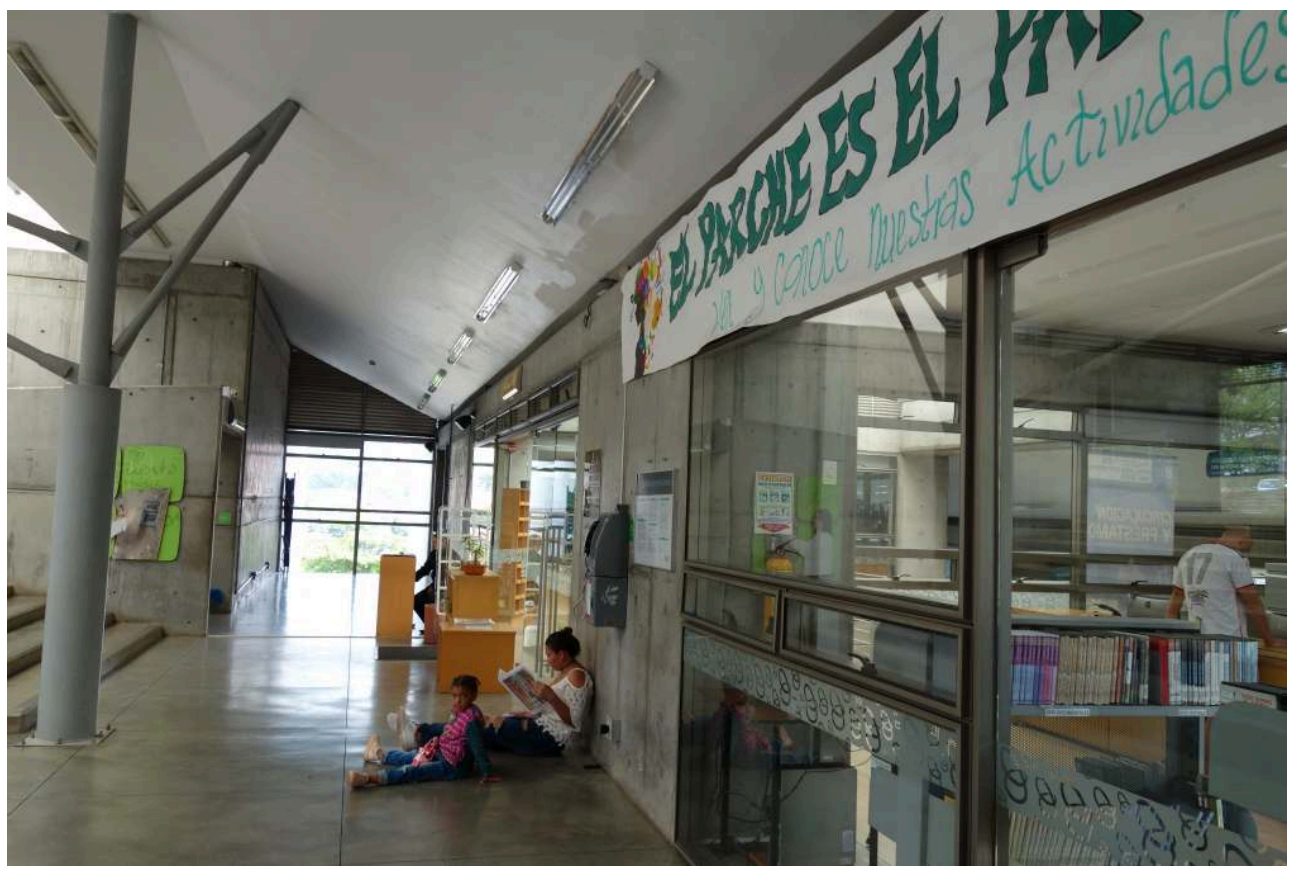

El Parque biblioteca San Javier fue inaugurado en 2006.

Autor, 2018

Los Parques Biblioteca como espacios dedicados a la cultura, evidencian que la intervención física va acompañada de la creación de escenarios de calidad que invitan a la participación ciudadana y la vinculación de diferentes sectores de la sociedad e instituciones públicas y privadas (Borja J., 2007; Alcaldía de Medellín, 2007). Esa intervención representó “cerca de 17000 metros cuadrados de salas de lectura, más de 56000 libros y material bibliográfico, colecciones bibliográficas generales, infantiles y especializadas; salones para talleres, salas para los artistas de los barrios cercanos, auditorios al aire libre y cerrados, salas de exposiciones, ludotecas, Centros de Desarrollo Empresarial Zonal -Cedezo-, 512 computadoras de uso libre conectadas a Internet con banda ancha, para el uso gratuito de la comunidad; cafeterías, papelerías, locales comerciales y más de 50000 metros cuadrados de espacio público, zonas verdes y recreativas" (Alcaldía de Medellín, 2007, p.153). Concebidos en gran parte como espacios puestos a la disposición de los propios habitantes, alcanzaron un gran éxito, lo cual detonó inclusive su fama mundial. Otro ejemplo muy destacable en la materia, en la misma ciudad de Medellín, es el Centro cultural Moravia, ubicado en un barrio marginado céntrico que albergaba un importante tiradero de basura a cielo abierto y que fue convertido en un parque. Diseñado por el gran arquitecto colombiano Rogelio Salmona, este centro cultural funge desde su inauguración en 2007 como una centralidad cultural y ciudadana mayor (bajo el concepto de "casa para todos"), en un barrio que se caracteriza todavía por su nivel elevado de pobreza y precariedad'. 
Figura 2. El Centro de Desarrollo cultural Moravia fue inaugurado en 2007 en el corazón del barrio popular que lleva el mismo nombre

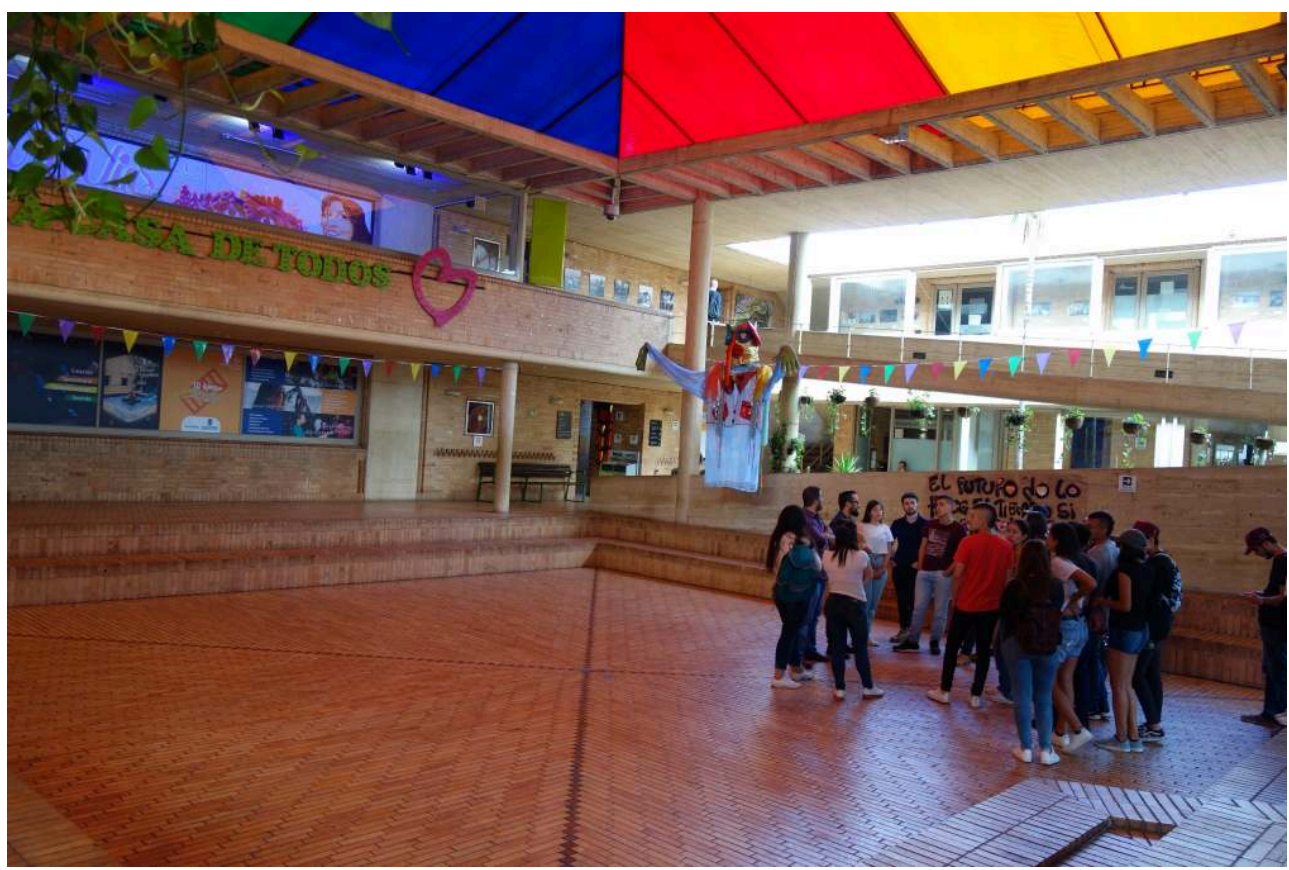

El Centro de Desarrollo cultural Moravia fue inaugurado en 2007 en el corazón del barrio popular que lleva el mismo nombre. Fue concebido para ser "un espacio para la convivencia, el desarrollo de la imaginación y la igualdad de oportunidades".

Autor, 2018

En la década del 2010, siguiendo el mismo modelo que consiste en dotar a los barrios populares con equipamientos culturales importantes, la ciudad de Medellín construyó unas veinte Unidades de Vida Articulada (UVA): aunque éstas constituyen más bien centralidades barriales enfocadas a la práctica del deporte, son en realidad espacios dedicados a la cultura en un sentido amplio, así como a la participación ciudadana, ya que albergan también a una amplia gama de actividades. La disponibilidad de los espacios ofrece la posibilidad de apropiarse del espacio a partir de la participación, así como de canalizar los usos del tiempo libre, potenciando cambios a nivel de la sociabilidad en las comunidades vinculadas.

Adicionalmente a esta política de construcción de grandes equipamientos culturales en el corazón de los barrios populares, la acción pública enfocada a la integración de estos últimos se centró en la rehabilitación de espacios públicos de calidad y la creación de otros nuevos, como elementos articuladores para las comunidades. Es así como la Municipalidad promovió la realización de murales por parte de los jóvenes de los barrios. La Comuna 13, conocida por su contexto de violencia y vulnerabilidad de las décadas anteriores, se ha vuelto un destino turístico internacional gracias a su graffitour, que permite conocer los murales que existen en el espacio público. Dicha actividad artística, legitima la acción pública de los ciudadanos de los mismos barrios, además de fomentar intervenciones que se plantean modificar las características identitarias de los mismos. 
Figura 3. En Medellín, los grafitis de la Comuna 13, realizados en el marco de las acciones de mejoramiento urbano del sector, se han vuelto un atractivo importante

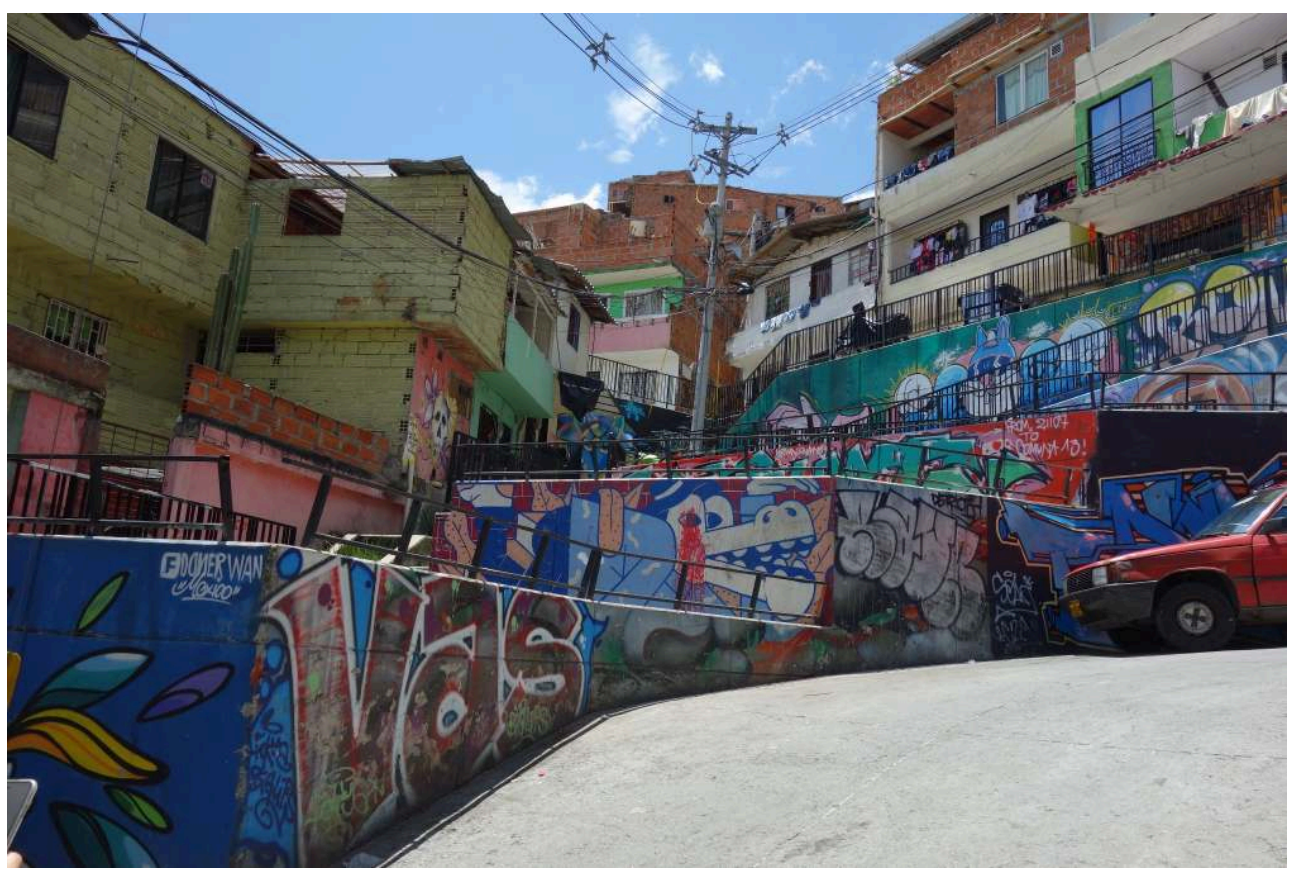

En Medellín, los grafitis de la Comuna 13, realizados en el marco de las acciones de mejoramiento urbano del sector, se han vuelto un atractivo importante.

Autor, 2018

Otra experiencia de mejoramiento de barrios que destaca en la región es el Programa de Recuperación de Barrios de Chile, también conocido como Programa Quiero mi Barrio. Desarrollado por el Ministerio de Vivienda y Urbanismo de Chile (MINVU) desde 2006, ha intervenido en 570 barrios en el país, todos ellos caracterizados por presentar deterioro físico y desintegración social (Ulriksen Moretti C., 2019). Su objetivo general es contribuir a mejorar la calidad de vida de los habitantes de barrios con problemas de deterioro, segregación y vulnerabilidad social, mediante un proceso participativo de recuperación de los espacios públicos y de los entornos de las familias.

Los criterios de selección para los barrios en que interviene este programa son el "deterioro urbano" y la "vulnerabilidad social". El primero remite a situaciones como la segregación urbana, el déficit y el deterioro del espacio público, la infraestructura y las instalaciones de la comunidad, la degradación del medio ambiente y a problemas de conectividad y accesibilidad. El segundo refiere a la situación de los habitantes: un débil sentimiento de pertenencia, estigmatización, deterioro de las relaciones sociales, inseguridad ciudadana y a la concentración de problemas en el ámbito de la educación.

El programa interviene sistemáticamente sobre el espacio público barrial, buscando mostrar al barrio como un espacio en el que se inscriben la sociabilidad, las experiencias y los recuerdos de todos. Mediante un trabajo conjunto y participativo con los habitantes, se intenta convertir al espacio barrial en una suerte de memoria en acción, en una realidad material que actúe sobre las conciencias individuales y estimule el cariño por el lugar, propiciando prácticas de apropiación y de orgullo (Campos Medina L. y A. Dupré, en prensa).

El programa suele emplear la realización de murales, como herramienta para activar la participación de los habitantes, y dejar un registro de la intervención en el espacio 
urbano, pero también actúa a través de la construcción de una historia de barrio. Se trata de una acción dirigida a la generación de un libro que recupere la historia de éste a través de encuentros cara a cara entre los habitantes y el equipo de intervención, y que convierta dicha historia en motivo de orgullo para los habitantes, de modo a revertir la dinámica de estigmatización que suele afectar estos barrios (Campos Medina L. y A. Dupré, en prensa).

Figura 4. Mural en Población Pedro Bannen, Lota, Región del Bío-Bío, Chile

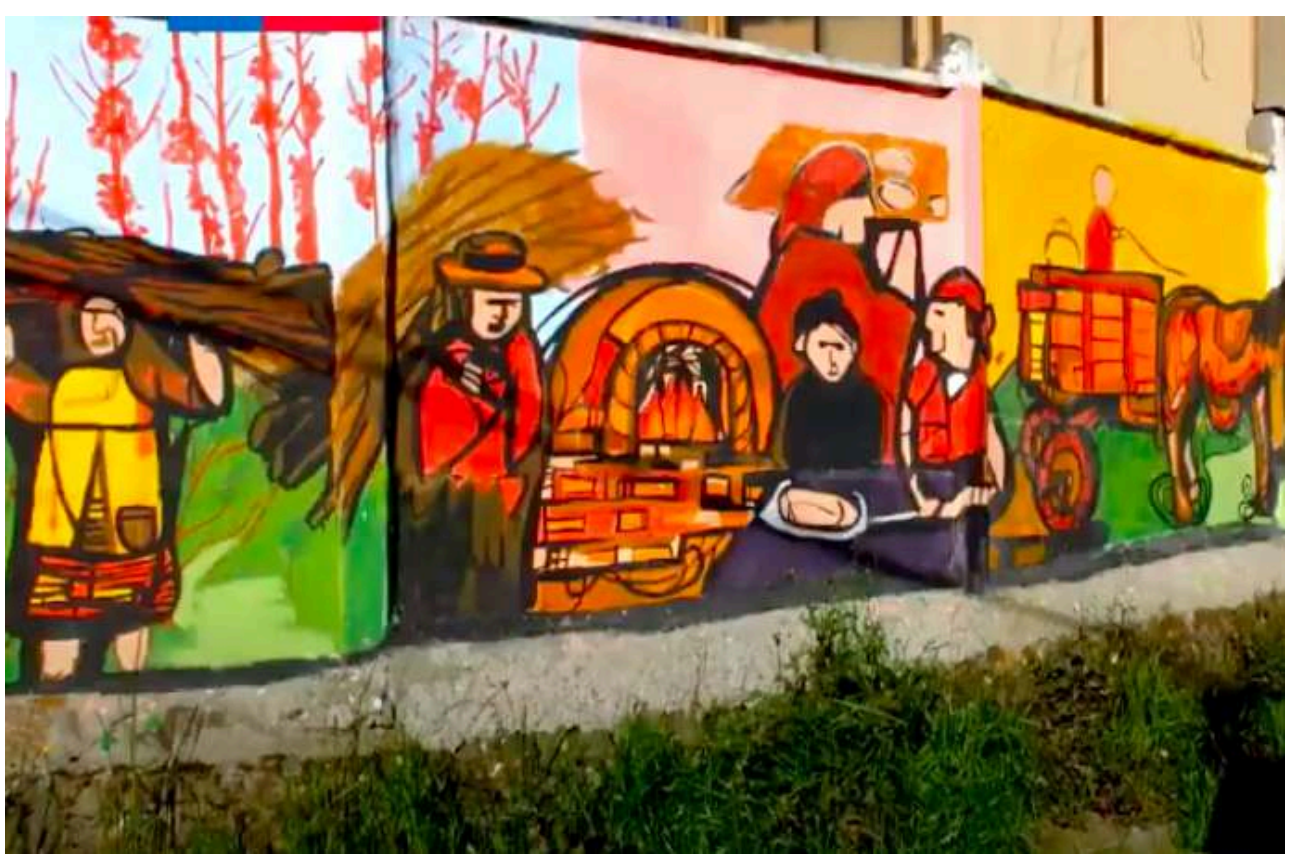

Mural en Población Pedro Bannen, Lota, Región del Bío-Bío, Chile.

Fuente: captura de pantalla de video del Ministerio de Vivienda y Urbanismo de Chile https:// www.youtube.com/watch?v=xDchiqrINug. Consultado el 4 de marzo de 2021.

Dentro de las numerosas iniciativas implementadas en la región, el Programa Comunitario de Mejoramiento Barrial (PCMB) de la Ciudad de México constituye otra experiencia destacable, cuya gran continuidad vale la pena subrayar, en un contexto generalmente marcado por el cese frecuente de proyectos al momento de los cambios de administración. Se inició en 2007 como iniciativa pública, con énfasis en los movimientos y organizaciones sociales y barriales de la ciudad. El programa propone como misión "capitalizar el talento, la imaginación y la creatividad de estos barrios para la transformación urbana"7. La ciudadanía, a través de sus organizaciones, presenta proyectos que son seleccionados por medio de un concurso y los elegidos reciben fondos para desarrollar sus propuestas directamente. El programa hace hincapié en la participación ciudadana, buscando relaciones de proximidad y corresponsabilidad entre el Gobierno y la ciudadanía, con el respaldo de organizaciones sociales, civiles y académicas asesorando las ejecuciones. La vinculación de diferentes actores es crucial en el desarrollo de este programa, ya que la principal fuente de financiación proviene del presupuesto del Gobierno de la Ciudad de México (hasta la reforma del 2017, Distrito Federal), además de considerar los aportes de las Alcaldías (antes de del 2017, delegaciones) y otras formas de financiación pública y privada (Mier y Terán A. et al., 2012). 

intervenciones en materia de mejoramiento de barrios. No obstante, ellas podrían globalmente ser categorizadas en tres grandes tipos, dependiendo de la naturaleza de las acciones de transformación urbana que conllevan. A pesar de sus diferencias, todas tienen en común el uso del arte y de la cultura como una herramienta relevante para desarrollar la integración social y la participación ciudadana, consideradas como claves para detonar la transformación urbana.

El PCMB constituye una iniciativa pública en barrios, colonias y pueblos de la Ciudad de México con altos grados de marginación, que propone la creación de espacios o recuperación de algunos abandonados a partir de la organización ciudadana. De esta forma se plantea la promoción y recuperación de espacios artísticos y culturales desde las propuestas de la ciudadanía, como dinamizadores del tejido social.

El programa se inserta en las políticas sociales urbanas, considerando las intervenciones artísticas dentro de la dimensión social y entendiendo que el rol activo que ejerce la ciudadanía implica la intervención desde su identidad, creación y formulación de socialización comunitaria a partir de las propuestas artísticas y culturales desarrolladas. Dicho énfasis social se reconoce en el tipo de obras realizadas en las 16 Alcaldías (ex Delegaciones), siendo los centros comunitarios, bibliotecas, salones de usos múltiples y casas de cultura los más frecuentes ${ }^{8}$. Lo anterior da cuenta de la prioridad que representan las actividades culturales comunitarias desde la perspectiva de la ciudadanía.

El PCMB se ha reconocido por el rol de la participación de la ciudadanía en las propuestas culturales, lo que es evidente en la evaluación desarrollada por Bazzaco y Sepúlveda (2010), en la que se identifica que la toma de decisiones comunitaria promueve actividades socioculturales. Además de reconocer que ocupan un lugar relevante para las comunidades, dada la demanda de los espacios para su uso y la necesidad de vincular características identitarias del barrio en las propuestas desarrolladas, es el caso del proyecto de títeres de la Colonia Morelos - Tepito centro oriente y la remodelación del Centro de artes y Oficios Escuelita Emiliano Zapata en la Colonia Pedregal de Santo Domingo

\section{Hacia una tipología de programas de mejoramiento de barrios usando el arte y la cultura como palanca para la transformación urbana y social}

\footnotetext{
Por una parte, nos encontramos con iniciativas de mejoramiento de barrio que se caracterizan por conllevar importantes ambiciones en el ámbito físico-espacial de escala urbana, como la provisión de infraestructuras en materia de servicios urbanos básicos (agua, energía, transporte público), la construcción de equipamientos e intervenciones de re-estructuración del tejido urbano. Es el caso de la intervención en barrios populares de la ciudad de Medellín, que constituye probablemente el ejemplo paradigmático en la materia (aunque se podrían mencionar otros como Favela Barrio, en Río de Janeiro o el actual Proyecto Barrio 31 en Buenos Aires). El uso del arte y la cultura constituye una dimensión importante de los programas, junto con las intervenciones físicas, que contribuye a potenciar.
} 

transformadoras claras pero cuyos ámbitos de actuación, a diferencia de los anteriores, se centran en intervenciones físicas acotadas a la rehabilitación de los espacios públicos y a acciones de fomento de la integración social en los barrios, a modo de un detonador de la transformación urbana. Los casos son también numerosos en la región y quedan bien ilustrados a través del Programa Quiero mi Barrio. Aunque se dan sobre todo en el ámbito de los barrios de origen informal, no se limitan a ellos, y existen también (cada vez más) iniciativas de este tipo en los barrios de vivienda social deteriorados con los que cuentan ciertos países, como es el caso de Chile y de México. 
mostrando variantes y énfasis relevantes de ser considerados a la hora de estimar los efectos que ellas han tenido a nivel de las dinámicas urbanas y sociales. No obstante, estas distinciones no deben ocultar un aspecto que resulta crucial, a saber, que mientras los grandes proyectos de regeneración urbana tienen al arte y la cultura como una consecuencia prevista y programada de su actuación, que se deriva de una fuerte intervención urbanística que modifica el tejido y la trama espacial de la ciudad, en los PMB el arte y la cultura emergen como el detonante, el agente causal principal de procesos de transformación social que, luego, buscan desencadenar una transformación urbanística.

En las diversas intervenciones que han servido de base para la interpretación aquí propuesta, es posible observar una lógica de actuación convergente que se caracteriza por enfocarse en el espacio público y convertirlo en elemento clave en la estrategia de intervención. Efectivamente, en el contexto de barrios deteriorados física y socialmente, donde las condiciones de habitabilidad son deficientes, la apuesta por el espacio público supone desplazar la atención hacia un objeto de propiedad común e implica ir a contrapelo de la idea de que el espacio público barrial ha perdido importancia como espacio de sociabilidad y como "estructura estructurante de comunidad" (Link F. et al., 2017: 11). Más aún, la intervención sobre los espacios públicos busca transformarlos en espacios de acción conjunta y desactivarlos en tanto productor de colisiones y conflictos, para así connotar al barrio como espacio en el que se plasma la sociabilidad, la identidad y el reconocimiento mutuo (Campos Medina, L. y A. Dupré, en prensa).

45 La condición de detonante que posee el uso del arte y la cultura se plasma en la intervención sobre los espacios públicos barriales, la que es efectuada de forma participativa y progresiva, generalmente involucrando el trabajo de las y los habitantes, de manera que se active una lógica de integración y se generen lugares de encuentro y convivencia, donde se pueda desarrollar actividades colectivas y comunitarias que potencien la identidad colectiva local. Se busca con ello aumentar el sentido de pertenencia y la satisfacción con los lugares de residencia y, sobre todo, evidenciar el rol jugado por las y los habitantes en el mejoramiento de la calidad de vida en contraste con las condiciones iniciales.

En este sentido, las diversas intervenciones aquí reseñadas comparten un mismo "referencial" (Müller 2002, 2010), vale decir, no sólo se asemejan en el conjunto de herramientas e instrumentos concretos para desarrollar la intervención (el repertorio de acciones artístico-culturales que efectúan), sino que también poseen una misma representación del fenómeno a intervenir, esto es, una imagen en función de la cual organizan su percepción del problema, examinan sus soluciones y definen sus propuestas de actuación. Esta representación o imagen está inspirada por similares principios, que en este caso remiten, esencialmente, a la integración y la cohesión social. Pero también comparten una similar "norma de cálculo" para realizar la intervención, es decir, convergen en entender que la intervención se justifica en aquellos barrios aquejados, conjuntamente, por problemas de deterioro físico y de descomposición social.

De esta forma, la inclusión del componente cultural en los PMB ha promovido el tránsito desde visiones sectoriales de la superación de la pobreza y la marginación, hacia intervenciones con lógica territorial que buscan generar cohesión e integración (Link F. et al., 2017; Matus C. et al., 2019; Maceratini E., 2013; Torres-Tovar C. et. al., 
2013). En general esto se corresponde con el paso de una lógica acotada de provisión de vivienda, hacia una lógica de integración social y urbana.

Sin embargo, pareciera ser que la mayor dificultad actual radica en la elaboración de criterios de evaluación que permitan estimar el grado de avance y consecución de ese objetivo de integración social y urbana, vale decir, evaluar de forma consensual cómo las intervenciones contribuyen a enfrentar las formas de deterioro físico y activar procesos de integración social en los barrios (Link F. et al., 2017). En esto, la situación latinoamericana se asemeja a lo ocurrido en Europa diez años atrás, en la medida que no se disponía de información que permitiera estimar con precisión los efectos de las intervenciones culturales realizadas. Por ello es que García (2008) planteaba la necesidad de realizar "estudios longitudinales que monitoreen la progresión de impactos y legados a largo plazo -más allá de los primeros cinco años” (p. 123).

Por otra parte, la concentración en los efectos de las intervenciones de los PMB sobre la sociabilidad parece esconder un problema importante, cual es la igualación entre, por un lado, integración social, y por otro, activación de la sociabilidad barrial. Ambos conceptos no son sinónimos, sino que responden a magnitudes diferentes. Aquí el modelo latinoamericano de intervención a través de programas de mejoramiento barrial se distancia de las intervenciones realizadas en Europa y Norteamérica en términos de regeneración urbana, cuyo efecto estructural pareciera ser mayor, en la medida en que involucra una transformación urbana y económica (García B., 2008).

En efecto, por más que se destaque la creciente articulación territorial de los PMB -que es un logro real de las intervenciones de los últimos años-, esto no significa una conexión estructural, es decir, una articulación entre las diferentes esferas comprometidas en la des-integración social de los barrios (Ruiz-Tagle J. y S. Romano, 2019), como son la provisión de servicios múltiples, la participación en el mercado del trabajo (Niembro A. et al., 2019) o el acceso al sistema educativo (Campos F., 2014). Este problema ha sido tematizado como la necesidad de integralidad en la intervención de los $\mathrm{PMB}$, la que emerge como el próximo desafío a afrontar y que involucra tanto la consideración integral de los territorios intervenidos, como el avance en la configuración de unidades de intervención de escala mayor a los barrios (Bustos M., 2016). Sin embargo, en esa reflexión, la dimensión cultural ha estado, hasta ahora, completamente ausente.

\section{Conclusión}

51 A pesar que se han llevado a cabo un gran número de programas de mejoramiento de barrios en Latinoamérica, existe todavía poca información referida a los contenidos de las intervenciones culturales en éstos $y$, por consecuencia, un déficit importante en la evaluación del impacto de las acciones realizadas en este ámbito. A modo de ejemplo, el caso de la transformación urbana en Medellín ha sido reconocido a nivel internacional, debido al aumento señalado de la participación y el mejoramiento de la comunicación comunitaria. Sin embargo, no se ha profundizado más acerca del peso del arte y de la cultura en la transformación urbana observada.

Si bien es cierto que la evaluación de este componente específico -en el contexto de intervenciones que son por excelencia multisectoriales- constituye una tarea altamente compleja9 ${ }^{9}$, contar con información sistematizada podría constituirse en un factor relevante en la comprensión de las dinámicas de integración social activadas en cada 
caso y en la promoción de procedimientos de evaluación más eficaces y adaptados a las intervenciones desarrolladas. El documento de Bazzaco y Sepúlveda (2010) da unas primeras aproximaciones para elaborar una metodología de evaluación desde la participación ciudadana presente en el desarrollo del PCMB en México. Además, considera como una de las variables a integrar el despliegue de intervenciones artísticas en proyectos urbanos. En un registro complementario, el trabajo desarrollado por el equipo Antesala (Ossandón F. et al., 2019) para el caso del Museo a Cielo Abierto de San Miguel, en Santiago de Chile, intenta esbozar los efectos e impactos del arte en los espacios públicos sobre la dinámica sociocomunitaria local.

Por otra parte, resulta necesario proponer nuevos marcos de análisis que den cuenta de la innovación que implica la inclusión sistemática del componente artístico y cultural en la intervención urbana, lo que se encuentra en sintonía con otras investigaciones que han argumentado en favor de incluir nuevas dimensiones en la consideración y análisis de las políticas públicas urbanas (Maceratini E., 2013; Torres-Tovar C. et al., 2013), particularmente aquellas relativas a la subjetividad y sociabilidad involucradas (Link F. et al., 2017; Marengo C. y A. Elorza, 2010; Matus C. et al., 2019), puesto que, como argumenta Aubán (2017: 81): "La introducción de la dimensión subjetiva como un elemento relevante del proyecto urbano llegará a convertirse en un factor decisivo del éxito o del fracaso de las políticas públicas de intervención urbana". El arte y la cultura han sido usualmente entendidos como potentes descriptores de esa dimensión subjetiva.

\section{BIBLIOGRAFÍA}

Alcaldía de Medellín, Del miedo a la esperanza, Medellín, Alcaldía de Medellín, 2007.

Aubán, Mónica, "La dignidad de los márgenes. Aproximaciones afectivas a la ciudad informal", Revista INVI, vol. 32, no 91, 2017, p. 67-89.

Auclair, Elizabeth, « Comment les arts et la culture peuvent-ils participer à la lutte contre les phénomènes de ségrégation dans les quartiers en crise ? ", Hérodote, vol. 3, n 69, p. 2006, p. 212-220.

Bellanger, Emmanuel, Collet, Anaïs, Desage, Fabien y Pierre Gilbert, « Rénovation urbaine. L'espace comme remède à la question sociale », Métropolitiques, 2018, https://metropolitiques.eu/ Renovation-urbaine-L-espace-comme-remede-a-la-question-sociale.html\#sommaire, page consultée le 4 mars 2021.

Brakarz, José, Ciudades para todos. La experiencia reciente en programas de mejoramiento de barrios, Washington D.C., BID, 2002.

Bazzaco, Edoardo y Sepúlveda, Sebastián, Barrio trabajando. Metodología de la evaluación de la Participación ciudadana, México, AECID, 2010. 
Borja, Jordi, "Revolución y contrarrevolución en la ciudad global: las expectativas frustradas por la globalización de nuestras ciudades”, Revista EURE - Revista De Estudios Urbano Regionales, vol. 33, no 100, 2007, p. 35-50.

Bustos, Mónica, “Áreas de interés para la gestión pública: aproximaciones para el diseño de una metodología de focalización territorial”, Revista INVI, vol.31, nº 87, 2016, p. 203-235.

Camargo, Luis-Miguel y Martin, Gérard, « Interventions urbaines et prévention des violences en Amérique latine : une analyse à partir des cas de Medellín et Fortaleza », Catherine Paquette (ed.), La ville durable, moteur de transformation sociale en Europe, Amérique latine et Caraibe, Paris, Institut des Amériques, 2019.

Campos, Fernando, "La escala geográfica de la exclusión en Santiago de Chile. Un análisis territorial de la política pública de regeneración barrial y mejoramiento de resultados escolares", Revista INVI, vol. 29, ํㅡ 82, 2014, p.65-89.

Campos Medina, Luis y Dupré, Amaëlle (en prensa), “El Programa Quiero mi Barrio como dispositivo de afectación sensible”, Bitácora Urbano Territorial.

Canto Chac, Manuel, “Gobernanza y participación ciudadana en las políticas públicas frente al reto del desarrollo", Politica y cultura, nº 30, 2008, p. 9-37.

Ceniceros, Brenda y Ettinger, Catherine, "Paisaje urbano desde la frontera Juárez- El Paso. Mapeando manifestaciones de arte urbano desde el bordo", Revista EURE - Revista De Estudios Urbano Regionales, vol. 46, nำ 137, 2020, p.181-201.

Córdoba-Hernández, Rafael y Pérez García-Burgos, Alonso, "Urbanización inclusiva y resiliente en asentamientos informales. Ejemplificación en Latinoamérica y Caribe”, Bitácora Urbano Territorial, vol. 30, no 2, 2020, p.61-74.

Decreto supremo nº 14, Reglamenta Programa Recuperación de Barrios, Santiago, Chile, 12 de abril de 2007, Recuperado de: https://www.leychile.cl/Navegar?

idNorma=259926ybuscar=recuperaci\%C3\%B3n+barrios, consultado el 15 de Marzo de 2021.

Desgouttes, Julie, « Les communs en friche ». Métropolitiques, 2019, https://metropolitiques.eu/ IMG/pdf/pdf_met_dossiercommuns_desgoutte.pdfpage consultée le 5 mars 2021.

Douay, Nicolas y Prévot, Marivonne, "Circulation d'un modèle urbain "alternatif" ? Le cas de l'urbanisme tactique et de sa réception à Paris ». EchoGéo, n 36, 2016, https://doi.org/10.4000/ echogeo.14617, page consultée le 4 mars 2021.

Duque, Isabel, "La cultura como estrategia de transformación y promoción urbana en Bogotá y Medellín”, Revista de Geografía Norte Grande, 61, 2015, p. 25-43.

García, Beatriz, "Política cultural y regeneración urbana en las ciudades de Europa Occidental: lecciones aprendidas de la experiencia y perspectivas para el futuro", RIPS. Revista de Investigaciones Políticas y Sociológicas, vol. 7, nº 1, 2008, p. 111-125.

García Canclini, Néstor, La sociedad sin relato. Antropología y estética de la inminencia, Buenos Aires, Katz Editores, 2016.

Giglia, Ángela, "Marginalidad, precariado y marginalidad avanzada: definiciones teóricas y realidades empíricas desde distintos contextos socio-espaciales en la ciudad de México", Territorios, $n^{\circ}$ 35, 2016, p. 59-80.

INFONAVIT, Memoria del Primer Foro Nacional de Regeneración Urbana de grandes conjuntos de viviendas sociales deteriorados, México, Instituto Nacional del Fondo de la Vivienda para los Trabajadores, 2020. 
Lerner, Jaime, Acupuntura urbana, Rio de Janeiro, Editora Record, 2003.

Link, Felipe, Greene, Margarita, Mora, Rodrigo, y Figueroa Martinez, Christian, "Patrones de sociabilidad en barrios vulnerables: Dos casos en Santiago, Chile", Bitácora Urbano Territorial, vol. 27, nº 3, 2017, p. 9-18.

Lusso, Bruno, « Les musées, un outil efficace de régénération urbaine ? Les exemples de Mons (Belgique), Essen (Allemagne) et Manchester (Royaume-Uni) », Cybergéo, 436, 2009 https:// doi.org/10.4000/cybergeo.21253, page consultée le 4 mars 2021.

Maceratini, Elisa, "Buenas prácticas de intervención integral en asentamientos informales", Bitácora Urbano Territorial, vol. 23, nº 2, 2013, p.85-93.

Matus, Christian, Ramoneda, Álvaro, y Valenzuela, Felipe, "La integración social como desafío: análisis del programa de campamentos en Chile (2011-2018)”, Revista INVI, vol. 34, no 97, 2019, p. 49-78.

Marengo, Cecilia, y Elorza, Ana, “Calidad de vida y políticas de hábitat. Programa de Mejoramiento Barrial en Córdoba, Argentina. Caso de estudio: barrio Malvinas Argentinas", Bitácora Urbano Territorial, vol. 17, nº 2, 2010, p. 79-94.

Masboungi, Ariella, Penser la ville par les grands événements, Gênes, Paris, Parenthèses Editions, 2011.

Mier y Terán, Arturo, Vázquez, Isabel, y Ziccardi, Alicia, “Pobreza urbana, segregación residencial y mejoramiento del espacio público en la Ciudad de México”, Sociologías, vol. 14, no 30, 2012, p. 118-155.

Ministerio de vivienda y urbanismo de Chile (MINVU). Recuperación de 200 barrios. Hacia la construcción de tipologías, Santiago de Chile, MINVU, 2010.

Niembro, Andrés, Guevara, Tomás, y Cavanagh, Eugenia, "Segregación residencial socioeconómica e inserción laboral: el caso de San Carlos de Bariloche, Argentina”, Revista INVI, vol. 34, no 97, 2019, p.129-154.

Paquette, Catherine, "Regeneración Urbana: un panorama latinoamericano", Revista INVI, vol. 35, no 100,2020, p.38-61.

Paquette, Catherine, Vivienda social y desarrollo urbano: contribución de la cooperación científica y técnica francesa a la problemática de la producción de vivienda social en México, Mexico, IRD/Service de coopération et d'action culturelle de l'Ambassade de France au Mexique, 2014.

Orozco Martínez, Yuriria, Hacia políticas de urbanización integral: los Programas de Mejoramiento de Barrios en América Latina, México, Centro de Estudios Sociales y de Opinión Pública de la Cámara de Diputados, 2015.

Ossandón, Fernando, Jara, Victoria, Poblete, Fernanda y Varas, Camila, “Impacto sociocomunitario del Museo a cielo abierto en los habitantes y la Villa de San Miguel”. 2019, https://museosacieloabiertolaantesala.files.wordpress.com/2019/10/informefinal_investigacic3b3n-macasam.pdf, page consultée le 4 mars 2021.

Rius, Joaquim, y Sánchez-Belando, María, "Modelo Barcelona y política cultural: usos y abusos de la cultura por parte de un modelo emprendedor de desarrollo local", Revista EURE - Revista De Estudios Urbano Regionales, vol. 41, nº 122, 2015, p.103-123.

Rius-Ulldemolins, Joaquim, y Posso Jiménez, Ladys, “Cultura, transformación urbana y empoderamiento ciudadano frente a la gentrificación. Comparación entre el caso de Getsemaní 
(Cartagena de Indias) y el Raval (Barcelona)", Revista EURE - Revista De Estudios Urbano Regionales, vol. 42, nº 126, 2016, p.97-122.

Ruiz-Tagle, Javier, y Romano, Scarlet, "Mezcla social e integración urbana: aproximaciones teóricas y discusión del caso chileno”, Revista INVI, vol. 34, nº 95, 2019, p. 45-69.

Rojas, Eduardo, Construir ciudades. Mejoramiento de barrios y calidad de vida urbana, Washington, Banco Interamericano de Desarrollo, 2009.

Torres-Tovar, Carlos, Rincón-García, John, Vargas-Moreno, Johanna, y Amaya-Medina, Natalia, "Hacer del Mejoramiento Barrial y Urbano una política pública estratégica para la superación de la pobreza", Bitácora Urbano Territorial, vol. 23, nº 2, 2013, p. 105-114.

Ulriksen Moretti, Constanza, “Genealogía del primer programa chileno de recuperación de barrios vulnerables "Quiero mi Barrio" en su primera generación 2006-2010", Revista INVI, vol. 34, no 96, 2019, p. 9-50.

UNESCO, Declaración de México sobre las políticas culturales. In conferencia mundial sobre las políticas culturales, México D.F., 26 de julio - 6 de agosto de 1982.

UNESCO, Universal declaration on cultural diversity. In adopted by the $31^{\text {st }}$ Session of the General Conference of UNESCO, Paris, 2 November 2001.

Vargas, Igrid y Jiménez, Eduardo, “Integración socio-espacial de asentamientos informales en Ibagué, Colombia. Un proyecto de cooperación al desarrollo”, Bitácora Urbano Territorial, vol. 23, no 2,2013, p. $117-128$

Vargas, Ingrid, Jiménez, Eduardo, Grindlay, Alejandro, y Torres, Carlos, “Procesos de mejoramiento barrial participativo en asentamientos informales: Propuestas de integración en la ciudad de Ibagué (Colombia)", Revista INVI, vol. 25, no 68, 2010, p. 59-96.

Zamorano, Mariano-Martín, "El modelo emprendedor de políticas culturales y el destination branding: un análisis a partir del filme Vicky Cristina Barcelona", Revista EURE - Revista De Estudios Urbano Regionales, vol. 46, nº 139, 2020, p. 91-112.

Ziccardi, Alicia, "Espacio público y participación ciudadana: El caso del Programa Comunitario de Mejoramiento Barrial de la Ciudad de México", Gestión y política pública, nº 21, 2012, p. 187-226.

\section{NOTAS}

1. Ver el Decálogo para el mejoramiento integral de barrios: http://onuhabitat.org.mx/ index.php/decalogo-para-el-mejoramiento-integral-de-barrios. Consultado el 4 de marzo de 2021.

2. Ver al respecto, para el caso de Francia, «Art et rénovation urbaine : la voie des possibles», $\mathrm{n}^{\circ}$ 69 des Cahiers $d u$ développement social urbain. https:/www.labo-cites.org/system/files/ documents/publications/2019-07/ExtraitsC69web.pdf. Consultado el 4 de marzo de 2021.

3. Https://issuu.com/ciudademergente_cem/docs/ut_vol3_2013_0528_10/55. Consultado el 4 de marzo de 2021.

4. Desde este paradigma la cultura dista de la noción relativa al arte y herencia en un grupo social, sino que se concibe como un activo económico, el núcleo del desarrollo urbano de carácter comercial (García B., 2008).

5. Ver por ejemplo:

https://www.fundacionhogares.org/documentos/EvalPintMexnov2016.pdf. Consultado el 4 de marzo de 2021. 
https://museosacieloabiertolaantesala.wordpress.com/tag/espacio-publico/. Consultado el 4 de marzo de 2021.

6. Http://www.oas.org/en/sedi/dsd/Biodiversity/Sustainable_Cities/Sustainable_Communities/ Events/Courses/Presentations/Day\%204/Centro\%20Cultural\%20Moravia.pdf. Consultado el 4 de marzo de 2021.

7. Mejora tu barrio CDMX. Página web oficial. Recuperado el 26 de agosto de 2020 de: https:// mejoratubarrio.com/el-programa-de-mejoramiento-barrial-y-comunitario/

8. García (citada en Bazzaco E. y S. Sepúlveda, 2010) agrupó en 11 tipologías las acciones desarrolladas por el PCMB en los primeros tres años de ejecución. El 44\% de las acciones involucran transformación de espacios comunitarios destinados a la vinculación en espacios culturales, a saber, más del 16\% en la construcción de Centros comunitarios (Casas de cultura, bibliotecas, salones múltiples), más del $15 \%$ en la rehabilitación de áreas comunes o áreas verdes, cerca del 9\% se destinó a rehabilitación y construcción de espacios deportivos y el 4\% para plazas públicas. Por otro lado, el casi $40 \%$ de las acciones fueron destinadas a luminarias, mobiliario urbano, arreglo y pintura de fachadas y puertas al exterior.

9. Un trabajo reciente sobre Medellín insistió sobre la gran dificultad con la que nos encontramos para atribuir los cambios sucedidos (en este caso, la disminución de la violencia) al Urbanismo Social, siendo muy numerosos los factores que influyen en el clima social y urbano de una ciudad, mucho más allá del tipo de acción pública llevada a cabo y por más que ésta haya sido exitosa (Camargo L. M., y G. Martin, 2019).

\section{RESÚMENES}

América Latina posee una tradición importante en materia de programas públicos de mejoramiento urbano de barrios marginados. Estos programas han conocido una evolución notable a lo largo del tiempo, pasando de ser iniciativas orientadas ante todo hacia la provisión de servicios urbanos básicos y el mejoramiento habitacional, a enfoques cada vez más multisectoriales poniendo mucho énfasis en la participación ciudadana y la coproducción de los proyectos con la población residente. En este contexto, el uso del arte y de la cultura ha cobrado una importancia creciente, como una palanca para la transformación urbana. Cuando los grandes proyectos de regeneración urbana implementados en las áreas centrales de las ciudades o zonas de baldíos en proceso de recuperación tienden a recurrir al arte y la cultura como una vía para lograr una mayor competitividad urbana y atraer nuevos residentes e inversionistas, los programas de mejoramiento de barrios marginados usan este recurso de manera muy diferente, como agente detonador de la integración social de los habitantes, a través de iniciativas que buscan activar y mantener su participación permanente y así, indirectamente, provocar transformaciones en el tejido urbano.

El objetivo del artículo es abrir la reflexión acerca de los rasgos característicos del uso del arte y la cultura en estos programas de mejoramiento de barrios marginados de América Latina, para entender la especificad del enfoque que materializan hacia la problemática que buscan atender, y contribuir así a la discusión acerca de una temática, hasta el momento, poco estudiada.

L'Amérique latine possède une tradition importante en matière de programmes publics d'amélioration de quartiers marginalisés. Ceux-ci ont connu une évolution notable au fil du temps. Les initiatives, d'abord avant tout centrées sur la dotation de services urbains essentiels et 
l'amélioration des logements, sont progressivement devenues multisectorielles, avec un fort accent mis sur la participation citoyenne et la coproduction de projets avec les habitants. Dans ce contexte, l'usage de l'art et de la culture comme un levier de transformation urbaine est devenu de plus en plus important. Mais alors que les grands projets de régénération urbaine mis en œuvre dans les zones centrales des villes ou les secteurs de friches urbaines en cours de récupération ont tendance à recourir à l'art et la culture dans le but de développer la compétitivité urbaine et d'attirer de nouveaux résidents et investisseurs, les programmes d'amélioration des quartiers marginalisés mobilisent cette ressource d'une toute autre manière, comme un agent déclencheur et un vecteur d'intégration sociale des populations résidentes, à travers des projets qui cherchent à activer et maintenir une participation permanente et engendrer ainsi, indirectement, une transformation de l'espace urbain.

L'article vise à ouvrir la réflexion concernant le recours à l'art et à la culture dans ces programmes d'amélioration des quartiers marginalisés en Amérique latine, dans le but de comprendre la spécificité de l'approche des problématiques urbaines qu'ils matérialisent et de contribuer à la discussion sur un sujet jusqu'à présent peu étudié.

Latin America has an important tradition of marginalized neighborhood improvement programs. These have undergone a significant evolution over time, shifting from initiatives focusing mainly on the provision of basic services and housing improvement to multisectoral projects giving high priority to citizen participation and to the co-production of urban actions with the resident population. In this context, the use of art and culture as a lever for urban transformation has become increasingly important. But when large urban regeneration projects implemented in city cores or wasteland areas in progress of recovery tend to use art and culture as a way to achieve greater urban competitiveness and to attract new residents and investors, poor neighborhood upgrading programs use this resource in a very different way, as a triggering agent for the social integration of the inhabitants, through project initiatives that seek to activate and maintain their permanent participation and thus, indirectly, generate an urban transformation of the site.

The article aims to initiate reflection on the use of art and culture in marginalized neighborhood improvement programs in Latin America, trying to understand the specific nature of their approach to urban issues and thus, contribute to the discussion on a topic that is still little explored.

\section{ÍNDICE}

Mots-clés: Programmes d'amélioration de quartiers, Art, Culture, quartiers périphériques marginaux, Amérique latine.

Keywords: Neighborhood improvement programs, Art, Culture, marginalized neighborhood, Latin America

Palabras claves: Programas de mejoramiento urbano, arte, cultura, barrios marginados, América Latina

\section{AUTORES}

\section{LUIS CAMPOS}

Profesor-Investigador. Instituto de la Vivienda (INVI), Facultad de Arquitectura y Urbanismo, Universidad de Chile, Portugal 84 Santiago, Chile. luiscampos@uchilefau.cl 


\section{CATHERINE PAQUETTE}

Investigadora. Centre d'Etudes en sciences sociales sur les mondes africains, américains et asiatiques, Institut de recherche pour le développement (IRD) / Université de Paris / INALCO.

Université Paris Diderot Case courrier 701775205 PARIS CEDEX 13. catherine.paquette@ird.fr 